\title{
Exploring Trends in the Global Small Satellite Ecosystem
}

\section{Jonathan R. Behrens and Bhavya Lal}

Science and Technology Policy Institute, Institute for Defense Analyses, Washington, District of Columbia.

\section{ABSTRACT}

Activity in the small satellite industry, especially since 2012, has grown as new organizations and nations have joined the evolving global market in both the private and public sector. Growth in the sector has been driven, in part, by growing capabilities and falling costs of smallsats, principally enabled by the miniaturization of technology for the small satellite platform, increased data processing capabilities, the ubiquitous presence of GPS enabling location and attitude determination, improvements in ground system costs and signal processing capabilities, and, finally, the deployment of relatively less expensive commercial off-the-shelf parts. This study characterizes the growing global small satellite ecosystem and the drivers that are currently influencing its evolution, through a scenario-based approach. After an initial survey of current activities in the sector, through the development of a database of more than $650 \mathrm{global}$ actors, and interviews with 70 experts across the sector in both the United States and internationally, four provocative archetypical scenarios were developed, set 10-15 years from now: There are two or more large small satellite constellations in low Earth orbit (LEO); small satellites have achieved near-parity with larger satellites, at least in remote sensing. Because of overcrowding and debris concerns, it is unsafe for satellite operation in LEO orbits; and on-orbit servicing, assembly, and manufacturing of spacecraft is a reality, decreasing the need for small satellites. An analysis of the four scenarios led to the identification of 62 drivers that could lead to each scenario. The drivers fell into four categories: (1) demand for LEO-based services, which drives availability of funding, technology development, development of low-cost approaches, and infrastructure; (2) access to space; (3) competing alternatives to space-based services; and (4) government policies. Although it is difficult to predict where the sector will be in 10-15 years, the sector will continue to be shaped by the interplay of governmental and commercial activities and changing policy regimes at the nationstate and international level. To examine where the smallsat sector is headed, it is important for policymakers to pay attention to drivers outlined in this and other studies.

Keywords: small satellite, CubeSat, commercial space, NewSpace, global trends

\section{INTRODUCTION}

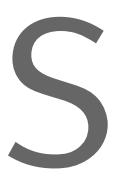

mall satellites (smallsats) are an emerging class of spacecraft, inclusive of platforms such as the CubeSat. Smallsats incorporate recent software and hardware improvements, most notably ones derived from the IT and electronics industries, and benefit from the resulting high capability that is feasible in small packages. ${ }^{1}$

Compared with traditional satellites, smallsats typically have shorter development cycles, smaller development teams, and, consequently, lower cost, both for the development and for the launch of the satellites. CubeSats, a class of smallsats, have the additional benefit of containerization, and a standardized form-factor, enabling mass production and easier launch vehicle integration, which can further lower cost. ${ }^{2}$ These lower-cost satellites' expendability, faster refresh, and simultaneous deployment in large numbers enable spatially or temporally distributed data collection-leading to greater risk-taking, experimentation, and creation of new applications that are not feasible with larger satellites. As a result, smallsats are making inroads in almost every area of space, including communication, remote sensing, technology demonstration, and science and exploration.

The satellites are operated by an ever-growing number of users. The global consultancy Euroconsult predicts that although fewer than a thousand smallsats were launched from

Manuscript presented at the 68th International Astronautical Congress, Adelaide, Australia, September 25-29, 2017. Copyright (C) Institute for Defense Analyses; 2019.

(c) IDA; Behrens and Lal, 2019; Published by Mary Ann Liebert, Inc. This Open Access article is distributed under the terms of the Creative Commons Attribution Noncommercial License (http://creativecommons.org/licenses/by-nc/4.0/) which permits any noncommercial use, distribution, and reproduction in any medium, provided the original author(s) and the source are cited. 
2006 to 2015 , up to 7,000 smallsats are likely to be launched between 2018 and 2027 for a variety of missions. ${ }^{3}$ Most of these satellites, $82 \%$, are associated with the roll-out of constellations planned by various companies, such as SpaceX and Boeing.

Given the growing perception that smallsats may enable new capabilities relative to existing space-based assets, a study was undertaken to identify global trends that would drive the smallsat sector in the coming decade. This article summarizes the study. ${ }^{1}$

\section{METHODOLOGY}

There is no universally accepted definition of a smallsat. Various groups and reports have classified smallsats according to their mass, volume, cost, capabilities, or some combination thereof. ${ }^{4-6}$ In our study, smallsats are defined as satellites with masses lower than $200 \mathrm{~kg}$ (with some exceptions up to $500 \mathrm{~kg}$ ). Small satellites include CubeSats, special types of cuboid-shaped smallsats that weigh between 1 and $10 \mathrm{~kg}$ and are created in units of a $10 \times 10 \times 10 \mathrm{~cm}$ cube.

With this definition of a smallsat, a scenario-based approach was developed to investigate the interaction between key factors and drivers that may affect developments in the smallsat ecosystem over the next $10-15$ years (i.e., 2,027-2,032). Scenario-based methodologies have been used across disciplines to inform policy, business, and academic endeavors-especially when projecting into an uncertain future. ${ }^{7,8}$

The authors recognize the diversity of approaches available under the umbrella of scenario methodology. The study employed an approach adapted from a four-phase process proposed in a meta-analysis conducted by the Institute for Futures Studies and Technology Assessment. ${ }^{9}$ The steps, in the context of this study, are as follows:

1. First, to understand the current state of the small satellite value chain, a database of more than 650 actors in the smallsat ecosystem was developed based on expert interviews and a literature review.

2. Next, key external as well as internal trends and forces driving the evolution of the small sat value chain were identified through semi-structured interviews conducted with 67 experts in government, industry, academia, and the finance community, as well as a review of the publicly available literature, industry publications, and government reports.

3. Informed by the trends and forces identified, a set of four hypothetical scenarios were designed to illustrate distinct potential end states (10-15 years into the future), with significant implications for the composition and direction of the global smallsat ecosystem. End states were chosen not for their likelihood but rather for their impact on future global policy regimes.

4. Finally, the drivers that could lead to one or more of these scenarios were identified. The near- and far-term trends in each were examined to the most feasible pathway, a cross-section of drivers that leads to a specific end state, for each scenario.

This article will focus on the results of steps 3 and 4. Further details on the analyses conducted on steps 1 and 2, which informed the information contained in this article, can be found in greater detail in reference 1.

\section{SCENARIOS}

Four scenarios, feasible in the timeframe of 2,027-2,032, were developed. These scenarios were chosen for their unique policy considerations. The four scenarios are not necessarily mutually exclusive; however, a combination of any number of drivers could pave a pathway to the scenarios. The drivers enabling each scenario, and more broadly influencing the smallsat ecosystem over the upcoming decade, will be discussed in detail in the Findings: Scenario Drivers section. Specific pathways to each scenario, and their likelihood, will be assessed in the Discussion: Likelihood of Pathways to Scenarios section.

\section{Scenario 1: Two or More Large Smallsat Constellations in Low Earth Orbit}

In this scenario, both broadband and imagery constellations of 100 or more small satellites are commercially successful in low earth orbit (LEO).

One or multiple broadband "megaconstellations" provide affordable global broadband internet with low latency. For example, terabits of throughput at $\$ 20 / \mathrm{Mbps} /$ month and 25-35 millisecond latency are delivered by a smallsat constellation. This compares against gigabits of throughput at $\$ 25 / \mathrm{Mbps} / \mathrm{month}$, and 500 millisecond latency from geostationary orbit (GEO)-high throughput satellites (HTS) existing in 2017. ${ }^{10}$

The imagery constellation provides affordable nearubiquitous optical imagery that refreshes at least once per hour with low resolution and an on-demand video of target areas. Costs have decreased by 1 to 2 orders of magnitude relative to 2017, and image resolution has reached at or below $1-5 \mathrm{~m} .{ }^{1}$

These large LEO constellations of smallsats co-exist with other ground, aerial, and space-based platforms, with respect 
to both communications (e.g., satellites in other orbits, terrestrial fiber, hot air balloons, solar internet planes, and airplane networks) and imagery (e.g., satellites in other orbits, payloads on permanent space stations, traditional aerial methods, and new unmanned aerial vehicle [UAV] approaches).

\section{Scenario 2: Smallsats Near-Parity with Larger Satellites in Remote Sensing}

In this scenario, as a result of remote-sensing capabilities being available commercially and outside the United States, a growing number of countries have access to technology that is at near parity with large satellites in remote sensing.

Near-parity has been achieved, especially, in three areas of high interest. The first is high ground resolution $(0.5 \mathrm{~m})$ on optical imagery. The second is the emergence of synthetic aperture radar (SAR) on a smallsat platform. Services such as tipping-and-cueing provided by optical and SAR satellite pairs previously available only on larger platforms are now feasible from smallsat platforms. The final are situational awareness (SA) services such as radio frequency (RF) mapping, automatic identification system (AIS) use, weather monitoring, space-based space situational awareness (SSA), GPS-Radio Occultation, and Automatic Dependent Surveillance-Broadcast (ADS-B).

As a result of these capabilities being available commercially and outside the United States, a growing number of countries have achieved near-parity in remote sensing with spacefaring nations. This does not mean that all countries have the same capability, rather that enough capability is available at an affordable cost to allow countries to independently meet their socioeconomic and national security needs.

\section{Scenario 3: Unsafe for Satellite Operation in LEO Orbits}

In this scenario, as a result of the growing number of smallsats in LEO and one or more high debris-causing collisions, it is unsafe to operate satellites in orbits between 500 and 1,200 km without risking collision. As a consequence, LEO is no longer viable for widespread commercialization without government reimbursement. Further, smallsats are larger and more expensive for operation in different orbits. Smallsats operating in higher orbits have an increased cost to manufacture, given the need for radiation and higher power, and are more costly to launch and operate.

\section{Scenario 4: On-Orbit Servicing, Assembly,} and Manufacturing of Spacecraft a Reality

In this scenario, multiple persistent platforms in LEO and GEO are being used by governments and the private sector for on-orbit servicing, assembly, and manufacturing (OSAM). On-orbit space capabilities weaken the case for building and launching smallsats from Earth, though they do not eliminate the need for them.

The scenario implies that large satellites are cost competitive, and hosted payload platforms have become the norm. These platforms offer the satellite industry the flexibility to design, build, and deploy satellites that are best suited for a given application.

To advance scientific knowledge, the new platforms allow for new ways to explore the solar system and deep space. For example, the size and cost of building a high-definition space telescope (HDST) is more readily addressed through the onorbit production platforms. An HDST with a 12-m mirror could identify 30 exo-Earth candidates within its lifetime, helping draw meaningful conclusions about the prospects of biological life on other planets. ${ }^{11}$

\section{FINDINGS: SCENARIO DRIVERS}

An analysis of these four scenarios led to the identification of 62 drivers. The list is not definitive or comprehensive, rather it is a characterization of the most influential drivers for the scenarios examined. Table 1 illustrates a selection of the drivers that are the most relevant to each scenario. Some drivers are closely aligned, such as demand for imagery products and a network of ground stations, whereas others are not aligned across the scenarios.

The individual drivers fell into the four categories: demand for LEO-based services, which, in turn, drives availability of funding, technology development, development of low-cost approaches, and infrastructure; access to space; competing alternatives to smallsats; and government policies.

For each category of drives, an overview, current trends, and, finally, projected trends when possible are provided.

\section{Demand for LEO-Based Services: Availability of Funding} and Customers

Demand for low-cost, high-speed broadband is driven by increased capacity for enterprise data (e.g., retail, banking, energy, and resources), growth of governments in industrialized countries, and demand for low-cost broadband among individual consumers in less developed countries and rural areas who may not have access to the internet.

These market drivers are currently inspiring investment in smallsat-based LEO constellations (e.g., OneWeb, Boeing and SpaceX). Estimates for broadband demand vary, and some experts predict that satellite broadband capacity demand (served by both small and larger satellites in GEO, MEO, and LEO orbits) will grow at a compound annual growth rate 


\begin{tabular}{|c|c|c|c|c|c|}
\hline & Drivers (Vectors with Magnitude and Direction) & Scenario 1 & Scenario 2 & Scenario 3 & Scenario 4 \\
\hline \multirow[t]{5}{*}{ Demand } & Demand for broadband, backhaul, and mobility & Medium & None & Medium & Medium \\
\hline & Demand for low latency internet & High & None & Medium & Medium (negative) \\
\hline & Demand for imagery/analytic products & High & High & Medium & Medium \\
\hline & Demand for situational awareness & None & High & Medium & Medium \\
\hline & Demand for OSAM & None & None & None & High \\
\hline \multirow[t]{4}{*}{ Funding } & U.S. government & Low & Medium (negative) & Medium & High \\
\hline & Foreign government & Low & High & Medium & Medium \\
\hline & U.S. venture and equity capital & Medium & Medium & Medium & Medium \\
\hline & Foreign venture and equity capital & Medium & High & Medium & Medium \\
\hline \multirow[t]{8}{*}{ Technology } & High resolution optical imaging & Low & High & Medium & Medium \\
\hline & SAR & None & High & Medium & Medium \\
\hline & On-board processing & High & Medium & Medium & Low (negative) \\
\hline & Optical communications & High & Medium & High (negative) & Medium \\
\hline & Advances in miniaturization & Low & High & Medium & Low (negative) \\
\hline & Spectrum related technologies & High & Medium & High (negative) & Low \\
\hline & Propulsion system & Medium & Low & High (negative) & Low \\
\hline & De-orbital and orbital debris removal technologies & Medium & Medium & High (negative) & Medium \\
\hline \multirow[t]{3}{*}{ Low Cost approaches } & Reliability of COTS components & Medium & High & Medium & Low \\
\hline & Robotics and automation for satellite integration & High & Medium & Medium & High \\
\hline & Modularity and standardization & Medium & Medium & Medium & High \\
\hline \multirow[t]{4}{*}{ Infrastructure } & SSA system & High & High & High & High \\
\hline & Network of ground stations & High & High & Medium & Medium \\
\hline & In-space relays & High & Low & Medium & Medium (negative) \\
\hline & Low-cost ground communication technologies & High & Low & Medium & Low \\
\hline \multirow[t]{2}{*}{ Launch } & Availability of reliable launch alternatives & High & Medium & Medium & Medium (negative) \\
\hline & Price of launch & Low & High & Medium & High (negative) \\
\hline \multirow[t]{5}{*}{ Competing alternatives } & Terrestrial for broadband & High (negative) & None & Medium (negative) & Medium (negative) \\
\hline & Airborne for broadband & High (negative) & None & Medium (negative) & Medium (negative) \\
\hline & Airborne for remote sensing & High (negative) & Medium (negative) & Medium (negative) & Medium (negative) \\
\hline & Large satellites for communication & High (negative) & None & Medium (negative) & High \\
\hline & Large satellites for remote sensing & High (negative) & Medium (negative) & Medium (negative) & High \\
\hline
\end{tabular}




\section{BEHRENS AND LAL}

\begin{tabular}{|c|c|c|c|c|c|}
\hline & Drivers (Vectors with Magnitude and Direction) & Scenario 1 & Scenario 2 & Scenario 3 & Scenario 4 \\
\hline \multirow[t]{6}{*}{ Government policies } & Regulating spectrum & High & Medium & High (negative) & Medium \\
\hline & On-orbit regulations & None & Low & Low & High \\
\hline & STM regime & High & Medium & High (negative) & Medium \\
\hline & Debris mitigation standards & Medium & Medium & High (negative) & Medium \\
\hline & National security (e.g., ITAR) & Low (negative) & High & None & Medium (negative) \\
\hline & Protectionism/mercantilism & Medium & High & Low & Low \\
\hline
\end{tabular}

A vector and its corresponding shading indicates the qualitative impact (low, medium, high) that a driver has on increasing the likelihood of a scenario. Note that a scenario's overall likelihood of occurring is the product of all drivers discussed, not any specific one. If a driver is expected to negate the possibility of a given scenario, a "negative" direction is specified. If not otherwise specified, each driver is presented in the affirmative. For example, "demand for low latency internet" is classified as "high" under scenario one, indicating that an increased demand for low latency will have a high impact on increasing the likelihood of Scenario 1.

COTS, commercial off-the-shelf; ITAR, International Traffic in Arms Regulations; OSAM, on-orbit servicing, assembly, and manufacturing; SAR, synthetic aperture radar; SSA, space situational awareness; STM, space traffic management.

of 29\% through 2024, reaching more than three terabits of bandwidth ${ }^{12}$; however, proposed LEO constellations collectively could deliver almost ten times as much, if operational. This would lead to some LEO constellations either not deploying fully or failing entirely. Were this to occur, it would not be too dissimilar to the dot-com bubble of the $1990 \mathrm{~s}$ where many companies failed, leaving behind a small number of robust organizations, infrastructure, and a trained workforce.

Growth in the demand for satellite imagery is expected to come from nongovernmental actors that could use images and image-based analytics for agriculture, economic forecasting, urban planning, resource management, disaster monitoring, retail, maritime, and other applications. Analysts expect the smallsat imagery market to rise at a compound annual growth rate of $49 \%$, which could take the market from $\$ 15$ million in 2015 to $\$ 8.8$ billion by 2030 if growth continues. ${ }^{13}$ In addition, smallsat imagery is expected to capture a growing fraction of the global geospatial imagery analytics market that is expected to grow to nearly $\$ 100$ billion by 2030 .

Although nongovernmental demand appears poised to grow, it is currently too small to point to a clear trajectory. Success will also be dependent on whether commercial smallsat organizations are able to convert images and metadata into useful information for end-users (e.g., business insights and trends). Examples include Cape Analytics' (United States) use of satellite-based imagery (along with imagery from other sources) to underwrite property values, and Descartes Labs' (United States) aim to project agriculture crop yields from both large government satellites and smallsat operators such as Planet and BlackSky (United States).

Finally, SSA services may become more widely available from emerging smallsat companies. Traditionally, SSA is provided from large platforms to customers who are able to afford high costs, such as governments and large enterprises. The SSA revenues are predicted to grow with a compound annual growth rate of $21 \%$ in the next 10 years. ${ }^{14}$ SSA market growth is difficult to estimate given that no planned smallsat projects are commercially viable yet. There are at least five commercial smallsat constellations proposing to provide AIS payloads for maritime users, two of which would also support ADS-B; two firms expecting to provide RF mapping services; and two focusing on space-based SSA.

Even with a bifurcated smallsat supply base, investment in smallsat infrastructure is expected to grow. Some providers plan to serve a large number of customers, often enterprises or individuals, who would be satisfied with low-end capabilities as long as they are available at low cost; others focus on a small number of customers, typically governments, who would pay more for high-end capabilities due to other benefits, such as frequent revisit rates, which may not otherwise be feasible with larger platforms. Thus, as the cost and development time for smallsats continue to decrease, lower levels of capital investment will be required for this first set as compared with larger satellites, enabling commercial investors and foreign governments that have smaller space budgets than established space organizations (e.g., European Space Agency, China National Space Administration, NASA, etc.). 
From a global perspective, venture capital (VC) firms located in the United States dominate the space VC ecosystem, investing currently in smallsat infrastructure and technologies. VC dominance, however, does not necessarily reflect or predict U.S. dominance in the global smallsat sector, partly because funds are not restricted to just U.S.-based firms, and partly because these firms are expected to eventually sell their products and services globally.

Interviewees, in both start-ups and established companies, cited other foreign countries as future customers, indicating that domestic governments were reluctant to invest too heavily in them before they were able to demonstrate a technology. Such "watch and wait" policies pushed the commercial entities to take foreign incentives to grow their businesses.

Interviews provided anecdotal evidence that foreign governments are also encouraging (through subsidies and direct funding) innovative companies from other countries to set up manufacturing and research and development (RED) facilities in their countries (e.g., Planetary Resources in Luxembourg, unnamed European interviewee in India). Where private funding is lacking, such as in Japan, governments are providing venture funds. A sign of a growing customer and investor base abroad is that U.S. firms are setting up shop in other countries (e.g., Tyvak in Italy). A continuation of this trend into the future would enable an increasingly globalized, decentralized, and larger community of smallsat developers and users.

Demand for LEO-Based Services: Technology Development, Low-Cost Approaches, and Infrastructure

Many of the companies that the Science and Technology Policy Institute research team spoke to believe that, given technologies currently deployed terrestrially and in space, fundamental REtD breakthroughs are not required for commercial success in the smallsat sector. Numerous technologies in the smallsat sector are borrowed from adjacent sectors and adapted to space. An example includes Phase Four (United States), who is borrowing aspects of its chip and processing technology for its propulsion technology from cell phones. There are, nonetheless, still opportunities for growth and new applications that would be supported through investment in the following areas: miniaturized and standardized components, high-resolution optical imaging, SAR, high bandwidth communications and download (e.g., optical communications and on-board processing technology), high delta-v propulsion, de-orbit and active debris removal, and technologies/software to reduce radiofrequency interference.
Instead of investing in fundamental research, private sector organizations are investing to reduce cost or increase reliability (at a low cost) of components and systems. This is accomplished through investment to increase the performance and reliability of commercial off-the-shelf (COTS) components, the integration of satellites by robotic systems, the development of mass manufacturing, and the increased use of modularity and standardization. Examples include firms such as York Systems (United States), who are redesigning satellite buses to be low-cost and mass manufactured; firms such as NovaWurks (United States), who are developing modular reconfigurable satellites; and others such as OneWeb Satellites (United Kingdom), who are developing standardized satellites that can be produced in robotic factories by the thousands.

In addition, firms are using novel business models in an effort to reach new markets and decrease costs. Access to data from satellites is typically expensive because data download requires multiple and expensive ground stations around the world, limiting smallsat operators' opportunities for lowcost operations. Breakthroughs in both technology and new business models are beginning to address this challenge. Japan's Infostellar is developing an AirBnB-style timeshare model in which down-time from existing ground stations is purchased by the broker and resold to smaller operators who can then have access to ground stations at much lower costs than would be feasible otherwise. Norwegian giant Kongsberg Satellite Services is adapting its business model to provide smallsat operators a streamlined and standardized process to gain access to its ground infrastructure, previously built for large satellites, at a fraction of the cost.

As with SSA, smallsats enable infrastructure for their own benefit. As an example, Kepler Communications (Canada) and Analytical Space (United States) propose the use of smallsat constellations for information relay by using optical communications. On the ground, BridgeSat (United States) is planning to provide ground stations with optical capabilities.

Finally, availability of low-cost ground antennas and user terminals that are able to integrate multiple signals and to track satellites that move quickly in LEO is one of the major challenges for the provision of affordable broadband services by megaconstellations. Companies such as Kymeta (United States) and Phasor Solutions (United States) are developing low-cost phased array technology into flat panel antennas. In particular, Kymeta's metamaterial antennas, which can be low cost because they are expected to be produced on commercial television manufacturing lines, are expected to supplant large and 
expensive ground stations. These and other solutions could enable antenna embedment in mobile platforms (cars, cell phones), changing the paradigm of communicating with space-based assets.

\section{Access to Space}

Current options for dedicated smallsat launch are limited to rideshares as secondary payloads on rockets launching large satellites or carrying cargo to the International Space Station. These options impose restrictions in terms of integration and launch schedules, orbit destinations, and loss of flexibility with respect to subsystems in the small satellite.

The process of procuring launch is complex enough for smallsat operators that companies such as Spaceflight Industries (United States), ECM Space (Germany), TriSept (United States), Tyvak (United States), and Innovative Solutions in Space (Netherlands) have developed technology to safely include large numbers of smallsats as secondary payloads on large launchers. Some of these companies act as launch brokers, purchasing entire launches from organizations such as SpaceX and ISRO, to sell slots to individual smallsat operators, sometimes as part of manufacturing and turnkey operations contracts.

Over the next decade, the emergence of launchers for only small payloads could provide relatively reliable, fast, and dedicated access to a variety of orbits and planes. A total of 34 organizations were identified (13 U.S.-based and 21 non-U.S.based) to have public projects to develop small satellite launchers to LEO with reference payloads ranging between 40 and $500 \mathrm{~kg}$. Companies seek to reduce the constraints that result from catering to the needs of a large primary payload. However, only a few of the small launchers are likely to succeed due to the following challenges: inability to compete pricewise (per kg cost) with large launchers, failure of technology, inability to raise the funding needed for development and operations, or an oversaturated market and thus the inability to compete.

Many approaches are in play to reduce the cost of launch. They include untraditional rocket technologies, such as airbreathing engines by Reaction Engines (United Kingdom), balloon launches by Zero2Infinity (Spain), and air launch by Virgin Galactic (United States). Further, this is also achieved through production processes and operational processes, such as 3D printing, used by SpaceX (United States), Rocket Lab (New Zealand), Vector Space System (United States), and Rocket Crafters (United States), extensive use of COTS components (e.g., Cubecab, United States), or reusability.

\section{Competing Alternatives}

Terrestrial, airborne (e.g., UAV), or large GEO satellites that are making both incremental and breakthrough improvements could outpace the eventual capabilities of smallsats, providing services sooner or more affordably. A similar situation occurred in the 1990s with satellite telephony.

Airborne platforms, such as airplanes (e.g., Airborne Wireless Network), UAVs (e.g., Facebook), or balloons (e.g., Google Alphabet's project Loon experiment), are exploring hosting payloads that are capable of competing with smallsat broadband. The use of the UAV platform for imagery has proliferated, and sensor technologies similar to those deployed on smallsats have been flown on UAVs. UAVs can compete with LEO constellations only on a regional basis. It is possible that UAVs could tap into a satellite-based broadband network and deliver data in real time in concert with orbiting observation satellites to deliver higher resolution data for specific areas of interest. UAV networks may complement LEO ones rather than replacing them.

On the broadband front, advances in HTS technologies would significantly increase the capacity delivered at a lower cost by large satellites. Several companies are developing and deploying high-latency broadband (e.g., Viasat, United Kingdom; and Hughes, United States), mobility-oriented satellites (Inmarsat GX, United States), and data-oriented satellites (Intelsat EpicNG, United States; Yahsat, United Arab Emirates; and Avantis Hylas, United Kingdom). Smallsat LEO communication constellations hold advantages with respect to shorter development cycles, higher risk tolerance, quick replenishment, and low latency. On the imaging front, large satellites could be a threat to remote sensing small satellites if they can offer high revisit rates, as Digital Globe is planning with its WorldView-Legion that purports to offer high revisit rates $(40 \times$ per day for some locations when combined with other satellites).

Widespread cellular towers offering $5 \mathrm{G}$ network service and fiber optic infrastructure are likely to continue to dominate markets in cities. However, satellite broadband also has a potential role in a hybrid $5 \mathrm{G}$ network, as a backhaul. Terrestrial and space-based alternatives could be integrated together to deliver continuous connectivity. In the hybrid system, satellite networks can be used for backup options for wireless and terrestrial providers to avoid overflowing of their networks. Satellite broadband would fill the gap, for example, in rural and remote areas within developed countries as well as in remote and landlocked areas in countries under development where terrestrial alternatives either cannot reach or are too expensive to be developed. Also, a low-latency smallsat broadband constellation, if successful, could provide 
broadband choices to users in large cities where consumers have a few choices for competitive options for high speed services from fixed broadband providers.

\section{Government Policies}

Finally, government policies at both the nation-state and international level could directly or indirectly influence the evolution of the smallsat ecosystem and industry. The following are a collection of government policies of interest: spectrum allocation and availability, on-orbit regulation, debris management and regulation, national security, and mercantilism.

According to interviewees, spectrum availability is currently a minor challenge for future smallsat missions; however, this could change. A current bottleneck exists due to the insufficient collaboration and communication among current and future operators of smallsat constellations from the different countries, something that is required to avoid RF inferences. In the next 10-15 years, new technologies and policies are expected to be developed to aid in spectrum availability and interference avoidance. However, cooperation between satellite operators would need to increase, and radio frequency interference (RFI) avoidance rules might need to be enforced. If rule enforcement exists, increased resources might be needed to watch for unintentional RFI, which may need to be tracked as part of the current SSA system.

Currently, there is no comprehensive global or domestic on-orbit regulation regime. Although there are regulations related to launch and re-entry, spectrum, and remote sensing in the United States, for example, there are no regulations related to on-orbit activities such as rendezvous and proximity operations, space-based SSA, or RF mapping. Internationally, with more than 70 countries engaged in smallsat activities, consensus has not been reached and there are a few indicators that there will be a comprehensive global regime beyond the high-level dictates in the Outer Space Treaty. Although operators have expressed interest in developing "rules of the road" that would provide certainty to investors, there are concerns about burdensome regulations that could drive companies to move from country to country. Given that the timeline in which operators and policymakers function does not always align, and the significant effort involved in creating international community agreement, developing policy and regulations for the quickly evolving commercial space industry will be a challenge for the next 10 years.

Current guidelines on debris mitigation are not enforced (or waivers are frequently granted by governments), and are not tailored to smallsats, especially large constellations in LEO. There are collaborations, both multilateral (e.g., Inter-Agency
Space Debris Coordination Committee under the United Nations Office for Outer Space Affairs, European Space Agency, Permanent Committee on Space Debris within the International Academy of Astronautics) and bilateral (e.g., United States with Japan/India/Australia, U.S. Department of State and China) that work toward space debris mitigation. Some of the efforts are focused on smallsats. In the current regulatory environment, a comprehensive national strategy would be developed in the next decade, most likely if there is a mishap that affects a strategic government asset. Otherwise, given the low cost and short lifespan of smallsats, stopgap measures will likely be taken in the absence of an international effort.

In all countries, mercantilist policies (e.g., tariffs, lending for foreign customers, restrictive immigration), if enacted, may protect a nascent domestic smallsat ecosystem as it matures, but simultaneously threaten to spark trade wars across countries. In most countries, export control policies, designed to protect national security interests, would likely not significantly affect the smallsat sector because the supply base is broader, and it is easier to develop smallsats that use international components or systems. However, the perception of such policies being restrictive has an effect. Going forward, most governments may be motivated to promote their nations' smallsat sector not only for economic development reasons but also to maintain global pre-eminence in space.

\section{DISCUSSION: LIKELIHOOD OF PATHWAYS TO SCENARIOS}

A combination of numerous drivers outlined in Table 1 could pave a pathway to each scenario examined. Two pathways will be discussed for each scenario, based on the drivers with "high" and "medium" impact on each scenario, followed by a discussion of the potential for each pathway's realization.

\section{Scenario 1: Two or More Large Smallsat Constellations in LEO}

Common drivers for both pathways are demand for broadband, low-cost ground communication technologies (e.g., ground antennas, user terminals, etc.), availability of network of ground stations, availability, reliability and frequency of launch alternatives, and orbital debris mitigation standards. Adding to these drivers and depending on how the smallsats communicate with each other and with the ground, Pathway 1 would require advances in technologies for the efficient use of the spectrum and policies to ensure availability of the spectrum if smallsats communicate by using the RF bands and Pathway 2 would require the development of optical communications if smallsats use intersatellite links 
and communicate with the ground by using optical frequency bands.

The presence of smallsat constellations in LEO is feasible in the 2030 timeframe. The drivers composing each pathway show technical progress, industry investment, and low barriers without the need to rely on technological miracles (or high consequence, low probability events) to set the scenario in motion. Four factors lead us to believe that the scenario is likely to come to fruition: (1) growing demand for broadband, communications, and imagery data; (2) technology availability and the small satellite design philosophy that emphasizes lower costs, rapid turnarounds, and quicker replenishments; (3) relatively low cost of constellations, including manufacturing and launching; and (4) policy environment required, which is already somewhat favorable.

Although competition from other sources of broadband, including terrestrial, aerial, and GEO satellites, could still affect the success of these constellations, LEO constellations would provide additional benefits such as global low latency services. It is likely that not all of the planned broadband constellations would succeed. Similar risks from aerial platforms and large satellites could also affect the imagery market but may be less likely because of the additional benefits from satellite platforms such as ubiquity, variety of sensors, high revisit rates, and predictable scheduling. In any case, the expectation is that large smallsat constellations could provide services more affordably.

Scenario 2: Smallsats Near-Parity with Larger Satellites in Remote Sensing Enabling Global Near-Parity

Common drivers for both pathways are demand for situational awareness, funding from foreign sources (e.g., government, VC, and/or equity capital), and high-resolution optical sensors and SAR for smallsats. Adding to these drivers, Pathway 1 would require advances to reduce the cost of manufacturing and operating smallsats such as development of components miniaturization, availability of reliable COTS components, modularity and standardization of buses and payloads, and decreased price of launch. For Pathway 2, U.S. policies such as protectionism/mercantilism or national security restriction (e.g., International Traffic in Arms Regulations) could lead together with the common drivers to the realization of Scenario 2.

A scenario of smallsat reaching near-parity with larger satellites in remote sensing is likely to be feasible in 10-15 years from now. There are substantial advances in all three technology areas considered (optical ground resolution, SAR, and SA), costs continue to fall, and price of launch is expected to continue decreasing. Also, smallsat systems for applica- tions from disaster monitoring to sea ice monitoring off coastlines are increasingly available commercially, and this trend is expected to accelerate. As a result, countries no longer need homegrown development capabilities to operate in space and would be able to achieve near-parity with spacefaring nations with relative ease.

\section{Scenario 3: Unsafe for Satellites to Operate in LEO}

Common drivers for both pathways are the absence of an efficient SSA system, the absence of a global space traffic management (STM) regime, and the lack of appropriate orbital debris mitigation standards. Adding to these drivers, the realization of Scenario 1 or Scenario 2 could lead to Scenario 3 becoming a reality because of the physical congestion of the orbital bands around 800 and $1,110 \mathrm{~km}$ or because of RF interferences.

An LEO unsafe for smallsat operation is unlikely in the next 10-15 years even when Scenarios 1 and 2 are likely to become a reality. However, near-misses with strategic assets in space may lead to restrictions on operations in certain orbits. All stakeholders interviewed are aware of the need for an efficient SSA system, and efforts by the government and the private sector are underway to improve SSA. This could ameliorate the SSA challenge in the coming decade and may make the need of an STM regime less urgent. Further, new debris mitigation guidelines are being considered in international fora, adapted for novel needs, as stakeholders increase their commitment to safe operations in space.

\section{Scenario 4: OSAM of Spacecraft a Reality}

Common drivers for both pathways are demand for OSAM, funding from either the U.S. government and/or foreign governments, technology required to perform activities on OSAM platforms such as robotics and automation for satellite integration, modularity and standardization, an SSA system tailored to the needs of OSAM activities, and, finally, on-orbit regulations. Adding to these drivers, the lack of reliable and frequent options to access space or a high price for launch could lead to the realization of Scenario 4.

OSAM becoming a reality in the next $10-15$ years is an unlikely scenario. The field of OSAM of spacecraft is still in its infancy, with government investments under $\$ 50$ million a year, and private investments being significantly less than that. The main driver for Scenario 4 to become a reality is increased investment by government and commercial entities, by an order of magnitude or more, to make enough progress and enable sophisticated OSAM platforms, human-tended or robotic, in LEO or GEO. Though recent assessments of the community do not indicate interest to invest at these high 
levels, ${ }^{11}$ this paradigm could change if the national security space enterprise, for example, finds OSAM as cost effective to address future space-based capabilities of other countries that meet or surpass U.S. assets.

\section{SUMMARY AND CONCLUSIONS}

The smallsat ecosystem is an emerging sector that has rapidly developed since 2012. As discussed in this article, there are a variety of inter-related drivers that are influencing both the direction and the growth of the ecosystem. Although it is not possible to predict where the sector will be in 10-15 years, the sector will continue to be shaped by the interplay of governmental and commercial activities and changing policy regimes at the nation-state and international level.

Given trends in drivers, the authors believe that the probability of Scenario 1 coming to fruition is high. Demand for broadband and imagery is growing, with funding following; required technology is available or is expected to be in the near future; and infrastructure breakthroughs needed are minimal. There are several rideshare options for launch, and further availability from large launchers and on-demand launch is expected in the next decade. Although the price of smallsat launch is decreasing, for at least broadband constellations, lack of reduction in price is not a deal-breaker given that these companies are making their business cases by using today's launch prices rather than assuming reduced prices in the future.

Scenario 2 is also likely to be feasible. Incremental advances are being made in all three areas considered (ground resolution, SAR, and situational awareness), and costs at every step of the supply chain continue to fall. Companies currently involved in these areas are likely to receive appropriate U.S. government permissions to operate commercially, and the rest of the world may follow.

Scenario 3 is unlikely to happen, although near misses with strategic assets in space may lead to restrictions on operations in certain valuable orbits. Both technology and policy efforts to develop propulsion capabilities are underway for smallsats, to improve SSA systems, and to develop international guidelines for the long-term sustainability of outer space activities.

Scenario 4 is unrealistic for the timeframe of interest, not because of any technological limitations but currently because of low investment in the area. OSAM capabilities are currently in their infancy, and R\&D investment would need to increase significantly to see OSAM platforms emerge in GEO and LEO, something not indicated for the near future. Impetus for future investment is likely to come from private sector satellite manufacturers that wish to reduce cost or increase revenues by assembling or enhancing large satellites in space, though a future large space telescope funded by a government could speed technology development.

Given the trends identified, we recommend that policymakers pay attention to the following:

- Speed at which enterprise and consumer demand for communication and imagery products/services is materializing. Proxy measures include tracking the emergence of new funders and funds for smallsat activity; the emergence and success of new start-ups, and their business plans; disruptive developments, especially related to high delta-v propulsion, low-cost manufacturing, resolution of imagery, and big data analytics; and foreign investment in upstream technology.

- Rates at which costs of manufacturing and other system costs for constellations are falling.

- Whether global governmental policies related to spectrum allocation and management and regulations related to SSA and debris are aligned with emerging technologies, and being rolled out at a fast-enough rate.

- Developments in alternatives to LEO-based services, especially terrestrial networks and large HTS.

- Alternative means for access to space as opposed to cost reductions.

- Developments that lower the cost of data transmission from small platforms in space-low-cost mobile antennas, ground stations, and in-space relay stations.

Data-gathering effort along these dimensions would help policymakers understand how the sector is changing, identify which actors have which capabilities, and assess the risks to their own assets, both in space and on the ground. Although it may not be possible for the United States to hold a large technological advantage in space over the rest of the world, it can continue to hold a large information advantage by strategically monitoring how these drivers are changing. Although the U.S. government may not be able to directly influence or deter some technological advances in unfriendly countries, it can strategically develop its own assets to operate in a new regime of ubiquitous satellite services that are more robust than accidental or intentional data gathering by private companies. Such a strategy requires being informed on the state of the identified drivers. By continually monitoring these drivers, nations can be better prepared to navigate the next few decades of space development.

\section{ACKNOWLEDGMENTS}

The authors would like to thank all the experts interviewed in the course of their research. Special thanks are due to the 


\section{BEHRENS AND LAL}

IDA researchers Elena De La Rosa Blanca, Ben Corbin, Ellen Green, Alyssa Picard, and Asha Balakrishnan for their contributions to this study.

\section{AUTHOR DISCLOSURE STATEMENT}

No competing financial interests exist.

\section{REFERENCES}

1. Lal B, de la Rosa Blanco E, Behrens JR, et al. Global trends in small satellites. Institute for Defense Analyses, IDA Paper P-8638, 2017.

2. Achieving Science with CubeSats: Thinking Inside the Box (National Academies of Sciences, Engineering and Medicine). Washington, DC: National Academies Press, 2016.

3. Prospects for the Small Satellite Market (Euroconsult). 2018. www.euroconsultec.com/research/smallsats-2018-brochure.pdf Accessed September 4, 2019.

4. Science Technology Mission Directorate. About SSTP. NASA. https://www.nasa .gov/directorates/spacetech/small_spacecraft/smallsat_overview.html Accessed 2017.

5. Small Satellite Missions, Background Paper 9 (Third United Nations Conference on the Exploration and Peaceful Uses of Outer Space). 1998;5. www.unoosa .org/oosa/oosadoc/data/documents/1998/aconf.184/aconf.184bp9_0.html Accessed September 4, 2019.

6. Hicks M, Niederstrasser C. Small Sat at 30: Trends, Patterns, and Discoveries. Small Satellite Conference. 2016 https://digitalcommons.usu.edu/smallsat/2016/ TS3YearInReview/12 Accessed September 4, 2019.

7. Philip N. Scenario Development: A typology of approaches. OECD. 2006. www .oecd.org/site/schoolingfortomorrowknowledgebase/futuresthinking/scenarios/ 37246431.pdf

8. Kosow H, Gassner R. Methods of future and scenario analysis. German Development Institute. 2008. https://www.die-gdi.de/en/studies/article/methods- of-future-and-scenario-analysis-overview-assessment-and-selection-criteria Accessed September 4, 2019.

9. Behrendts S, Erdmann L, Nolte R, Diegner B. Integrated technology roadmapping. Institute for Futures Studies and Technology Assessment. 2007. https:// www.izt.de/fileadmin/downloads/pdf/IZT_WB87.pdf Accessed September 4, 2019.

10. HughesNet Gen5 Surpasses 100,000 Subscribers in Just Two Months (Echostar). 2017. http://ir.echostar.com/news-releases/news-release-details/ hughesnet-gen5-surpasses-100000-subscribers-just-two-months Accessed September 4, 2019.

11. Boyd I, Buenconsejo RS, Piskorz D, et al. On-orbit manufacturing and assembly of spacecraft. Institute for Defense Analyses, IDA Paper P-8335, 2017.

12. LEO HTS Constellations What Happens If (Northern Sky Research). 2015. www.talksatellite.com/Americas-A10085413.htm Accessed September 4, 2019.

13. Assessment of the Small Satellite Market (Frost and Sullivan). 2015. https:// store.frost.com/assessment-of-the-small-satellite-market.html Accessed September 4, 2019.

14. Small Satellite Markets, $3^{\text {rd }}$ Edition (Northern Sky Research). 2017. https:// www.linkedin.com/pulse/situational-awareness-key-smallsat-target-carolyn-belle Accessed September 4, 2019.
Address correspondence to: Bhavya Lal Science and Technology Policy Institute Institute for Defense Analyses 1701 Pennsylvania Avenue NW Washington, DC 20006 E-mail: blal@ida.org 